\title{
Studies on influence of genotypic diversity on yield, quality and incidence of white fly and yellow mite in Capsicum annuum $\mathrm{L}$.
}

\section{S. Datta* and G. Chakraborty ${ }^{1}$}

Department of Vegetable and Spice Crops, Uttar Banga Krishi Viswavidyalaya, Pundibari, Cooch Behar-736165 (West Bengal), INDIA

${ }^{1}$ AINP on Jute and Allied Fibre, Uttar Banga Krishi Viswavidyalaya, Pundibari, Cooch Behar-736165 (West Bengal), INDIA

*Corresponding author. E-mail: suchanddatta@gmail.com

Received:A pril 15, 2013; Revised received:J une 17, 2013, Accepted: August 12, 2013

Abstract: A field experiment was conducted with fifty one chilli (Capsicum annuum L.) genotypes to study the growth, yield, quality characters, white fly and yellow mite incidence during Rabi season of 2007-08 and 2008-09. The data on growth, yield performance, quality characters and pest incidence revealed significant variation among the different genotypes. Significantly the highest number of fruits per plant was recorded in genotype CA-29 (168.23) and it was lowest in genotype CA-2 (52.30) and it was statistically at par with genotypes CA - 15 (56.09) and CA-43 (56.20). Like number of fruits per plant, the significantly highest fresh yield was recorded in CA-29 (14.58 t/ha). Higher fruit yield was also recorded in genotype CA - 47 (13.35 t/ha) and CA-48 (13.18 t/ha). Ascorbic acid content in red ripe fruit varied from 75.89 to $167.21 \mathrm{mg} / 100 \mathrm{~g}$ fresh. The highest capsaicin content in the red ripe fruit was recorded in genotype CA -17 (1.13\%) followed by CA-4 (1.07\%) and it was lowest in CA-7 (0.33\%). Genotype CA - 45 was almost free from white fly incidence and lower incidence was also recorded in genotype CA-43 (0.40/plant) followed by CA - 23 ( 0.50 / plant) and CA - 21 (8.10/ plant) was found most susceptible to white fly incidence. Among the different genotypes, CA - 22, CA - 24, CA - 25, CA - 27 and CA - 30 were free from mite infestation where as CA - 13 (13.60/leaf) was most susceptible to yellow mite incidence.

Keywords: Chilli, Growth, Mite, Quality, White fly, Yield

\section{INTRODUCTION}

Chilli (Capsicum annuum L.) is one of the most valuable and commercial vegetable and spice crops of West Bengal as well as in India. The genus Capsicum originated in the American tropics. It is probably introduced by Portuguese into southern parts of India and cultivation spread out throughout India by the end of $19^{\text {th }}$ century. It is considered as one of the important cash crop in the northern parts of the West Bengal. Chillies are sold in local market or supplied to distant places as cash crop fetching a good return to the farmers. It is used for its pungency, colour and its spicy taste. Green chillies are also rich in vitamin A and vitamin C. The average dry chilli yield of the country is low as compared to the progressive chilli producing countries like USA, Korea and Taiwan (Prasad et al., 2003). Productivity of chilli in the Sub-Himalayan region is low (0.96 tonnes/ha) as compared to national level (1.2 tonnes/ha) (Deb et al., 2008). Among several factors, lack of improved varieties and incidence of different pest are the main constraints for getting higher production. Among the different pest of chilli white fly (Bemisia tabaci) is an important pest of chilli (Othman et al., 2002). White fly is basically polyphagous pest and the most preferred plant species belong to family, Solanaceae (Othman et al., 2002). It is found on the underside of leaves. Both nymphs and adults use their long mouthparts (stylets) to reach the phloem of the leaves and remove sap that is continuously excreted. The sweet substance ("honeydew") covers the plant parts where the whiteflies are feeding, providing a suitable substrate for some superficial fungi known as "sooty molds." B. tabaci can also transmit chilli leaf curl virus disease in a persistant manner (Thresh, 1974 and Brown, 1994). B. tabaci can rapidly disseminate viruses in the field even when populations are not appreciable, and cause severe crop damage in susceptible plantings. Yellow mite (Polyphagotarsnemus latus Bank) is also the serious pests infesting chilli (Amin, 1979). Both nymps and adults of mite affect the vigour and vitality of the crop. Affected leaves either curled downward due to mite called chilli leaf curl disease. The overall reduction in yield of dry chilli ranged from 40-70\% due to infestation of thrips and yellow mite (Jagadeesha et al., 2000). Several insecticides have been evaluated against white fly and yellow mite and reported to be effective. But the indiscriminate usage of insecticides to control these 
pests has resulted health and resurgence of pest (especially in case of mite) (Mondal and Mondal, 2012). Though a large number of varieties have developed from different research station but very few information is available for terai region of West Bengal. Considering these points, the present investigation was undertaken to identify the promising genotypes having higher yield and quality attributes and resistance and tolerance to white fly and mite.

\section{MATERIALS AND METHODS}

A field experiment was conducted at the experimental field of Uttar Banga Krishi Viswavidyalaya, Pundibari, Cooch Behar, West Bengal during the Rabi season of 2007-08 and 2008-09 to study the yield, quality performance, white fly and yellow mite incidence. Fifty one chilli genotypes (CA-1 to CA-51) studied in this experiment by adopting randomized block design with three replications. The climatic condition of this region is sub-tropical humid. The soil was sandy clay loam in texture having $\mathrm{pH} 5.6,0.90 \%$ organic carbon, $133.35 \mathrm{~kg} /$ ha total nitrogen, $45.21 \mathrm{~kg} / \mathrm{ha}$ available phosphorus and $59.94 \mathrm{~kg} / \mathrm{ha}$ potash.. Transplanting was done at $3^{\text {rd }}$ week of November in a plot of $3.60 \mathrm{~m} \times 3.0 \mathrm{~m}$ size with a spacing of $45 \mathrm{~cm} \times 30 \mathrm{~cm}$. Well rotten farmyard manure @ 15 tonnes/ha was applied as basal. Inorganic fertilizers were applied as $100 \mathrm{~N}: 50 \mathrm{P}_{2} \mathrm{O}_{5}: 50 \mathrm{~K}_{2} \mathrm{O} \mathrm{kg} / \mathrm{ha}$. Full dose of $\mathrm{P}_{2} \mathrm{O}_{5}$, one third of nitrogen and half of potash was given as basal at the time of land preparation. After 45 days of transplanting top dressing was done with one third of nitrogen and rest half $\mathrm{K}_{2} \mathrm{O}$. Second top dressing was done with rest one third of nitrogen at 75 days after transplanting. Irrigation, weeding and other cultural practices and diseases management were carried out accordingly at required stages. Both in nursery and main field no insecticide was sprayed. Observations were recorded on different morphological and yield attributing characters from five randomly selected plants per plot. Ascorbic acid in chilli was determined by colorimetric method as suggested by Ranganna (2001). Capsaicin content $(\%)$ of red fruits was measured by spectrophotometric method as described by Sadasivam and Manickam (1996). Leaf chlorophyll content was measured by cholorophyll meter (with the unit of SPAD502) at the time of first harvest. Observation for white fly and yellow mite were recorded from fifty days after transplanting upto the end of March at fifteen days interval and make it average. Mite incidence on the chilli plants themselves was counted on individual leaves taken from top, middle, and lower canopy and white fly population was counted on basis of whole plants from 10 randomly selected plants per plot. White fly remains inactive and reluctant to fly during early hours of the day. Hence, white fly populations were counted in between 6.30 am to 7.30 am (Saikia and Muniyappa, 1989). Later, these selected leaves are examined under stereobinocular microscope for counting the number of mites per leaf (Mondal and Mondal, 2012). Estimation of yellow mite was done on randomly selected plants. Each leaves was observed under a stebinocular microscope as described by Awate et al. (1981). Statistical analysis was done as per method suggested by Gomez and Gomez (1984).

\section{RESULTS AND DISCUSSION}

Perusal of the data presented in tables 1-3 revealed that there was a significant variation with respect to growth, yield and quality characters among the different genotypes.

G rowth, yield and yield characters: The significantly $(\mathrm{P}<0.05)$ highest plant height was recorded in genotype CA-43 $(105.20 \mathrm{~cm})$ and it was lowest in genotype CA-26 $(34.20 \mathrm{~cm})$. Plant height range of 34.20 to $105.20 \mathrm{cmrecoded}$ in this experiment differed from the findings of Chaudhary and Samadia (2004) under Bikaner condition and Khurana et al. (2003) under Ludhiana Punjab condition. This variation might be due to agroclimatic condition and variation of genotypes. The maximum number of primary branches (8.50) was recorded in genotype CA-29 which was statistically at par with genotype CA-30 (8.07). The highest plant spread was recorded in CA-18 $(56.60 \mathrm{~cm})$ and it was lowest in CA-23 $(37.43 \mathrm{~cm})$.

Among the different genotypes leaf chlorophyll content varied from 50.87 - 66.74 SPAD-502. Gogate et al. (2006) also reported that chlorophyll content among the genotypes varied significantly. Significantly the highest umber (168.23) of fruits per plant was recorded in CA-29. Higher number of fruits per plant was also recorded in genotype CA- 47 (153.97) followed by CA-28 (151.27) and CA-30 (149.47) and CA-48 (144.57). But it was lowest in genotype CA-2 (52.20), which was statistically at par with genotypes CA-15 (56.00) and CA-43 (56.20). Among the different genotypes significantly highest fresh fruit yield per hectare was recorded in genotype CA-29 (14.54 tonnes/ha). The higher fresh fruit yield per hectare was also recorded in genotypes CA-47 (13.35 tonnes /ha), CA-48 (13.18 tonnes/ha) and CA-30 (12.99 tonnes/ha). The lowest yield was recorded in genotype CA-43 (4.58 tonnes/ha) which was also statistically at par with CA-2 (5.74 tonnes/ha). The higher yield of genotypes CA-29, CA-47, CA-48 and other genotypes might be due higher number of fruits per plant. Hundal and Khurana (1988) reported that fruit yield in chlli varieties and hybrids varied from 0.23 tonnes per ha to 33.52 tonnes per ha. This finding support the observation on fresh yield of the present experiment.

Quality characters: Maximum ascorbic acid content $(167.21 \mathrm{mg} / 100 \mathrm{~g}$ fresh) was recorded by CA- 27 and it 
Table 1. Plant height, primary branches and plant spread of different chilli genotypes.

\begin{tabular}{|c|c|c|c|c|c|c|c|c|c|}
\hline \multirow[t]{2}{*}{ Genotypes } & \multicolumn{3}{|c|}{ Plant height $(\mathrm{cm})$} & \multicolumn{3}{|c|}{ Primary branch/plant } & \multicolumn{3}{|c|}{ Plant spread (cm) } \\
\hline & $2007-08$ & $2008-09$ & Pooled & $2007-08$ & $2008-09$ & Pooled & $2007-08$ & $2008-09$ & Pooled \\
\hline CA-1 & 64.03 & 75.87 & 69.95 & 5.33 & 6.33 & 5.83 & 44.10 & 47.80 & 45.95 \\
\hline CA-2 & 90.13 & 91.40 & 90.77 & 4.33 & 5.23 & 4.78 & 40.57 & 45.80 & 43.19 \\
\hline CA-3 & 75.13 & 86.27 & 80.70 & 5.53 & 6.20 & 5.87 & 45.03 & 43.27 & 44.15 \\
\hline $\mathrm{CA}-4$ & 86.93 & 95.20 & 91.07 & 6.07 & 6.27 & 6.17 & 40.23 & 46.367 & 43.30 \\
\hline CA-5 & 72.87 & 67.07 & 69.97 & 6.07 & 6.40 & 6.24 & 47.87 & 45.93 & 46.90 \\
\hline CA-6 & 73.80 & 85.47 & 79.64 & 6.07 & 5.67 & 5.87 & 45.13 & 48.47 & 46.80 \\
\hline CA-7 & 72.80 & 75.47 & 74.14 & 5.67 & 6.00 & 5.84 & 48.67 & 55.27 & 51.97 \\
\hline CA-8 & 79.27 & 89.33 & 84.30 & 6.00 & 6.13 & 6.07 & 51.30 & 50.63 & 50.97 \\
\hline CA-9 & 90.67 & 89.20 & 89.94 & 6.33 & 6.13 & 6.23 & 51.60 & 50.87 & 51.24 \\
\hline CA-10 & 83.67 & 88.53 & 86.10 & 5.67 & 5.87 & 5.77 & 47.73 & 52.13 & 49.93 \\
\hline CA-11 & 77.80 & 88.87 & 83.34 & 5.40 & 5.73 & 5.57 & 49.40 & 50.73 & 50.07 \\
\hline CA-12 & 93.70 & 89.07 & 91.39 & 5.53 & 5.97 & 5.75 & 51.23 & 53.00 & 52.12 \\
\hline CA-13 & 64.73 & 59.67 & 62.20 & 5.87 & 4.87 & 5.37 & 43.67 & 43.07 & 43.37 \\
\hline CA-14 & 104.87 & 87.60 & 96.24 & 5.93 & 6.03 & 5.98 & 54.47 & 56.37 & 55.42 \\
\hline CA-15 & 86.07 & 98.33 & 92.20 & 5.00 & 4.93 & 4.97 & 51.07 & 50.60 & 50.84 \\
\hline CA-16 & 82.67 & 77.66 & 80.17 & 6.87 & 7.20 & 7.04 & 55.00 & 55.53 & 55.27 \\
\hline CA-17 & 91.27 & 81.83 & 86.55 & 6.07 & 6.27 & 6.17 & 51.80 & 53.90 & 51.85 \\
\hline CA-18 & 84.83 & 92.87 & 88.85 & 5.20 & 5.60 & 5.40 & 55.47 & 57.73 & 56.60 \\
\hline CA-19 & 88.07 & 99.20 & 93.64 & 5.13 & 6.00 & 5.57 & 53.27 & 56.67 & 54.97 \\
\hline CA-20 & 83.60 & 87.53 & 85.57 & 6.80 & 6.73 & 6.77 & 50.97 & 50.23 & 50.60 \\
\hline CA-21 & 75.27 & 87.53 & 81.40 & 6.47 & 6.40 & 6.44 & 51.50 & 49.70 & 50.60 \\
\hline CA-22 & 83.67 & 87.40 & 85.54 & 7.53 & 7.33 & 7.43 & 52.37 & 51.80 & 52.09 \\
\hline CA-23 & 89.87 & 84.87 & 87.37 & 5.87 & 5.20 & 5.54 & 35.53 & 39.33 & 37.43 \\
\hline CA-24 & 67.80 & 63.87 & 65.84 & 5.73 & 5.83 & 5.78 & 47.53 & 52.17 & 49.85 \\
\hline CA-25 & 91.80 & 74.60 & 83.20 & 5.40 & 5.73 & 5.57 & 45.43 & 47.50 & 46.47 \\
\hline CA-26 & 34.07 & 34.33 & 34.20 & 5.47 & 5.40 & 5.44 & 47.80 & 50.80 & 49.30 \\
\hline CA-27 & 104.73 & 93.47 & 99.10 & 6.53 & 6.70 & 6.62 & 55.27 & 57.00 & 56.14 \\
\hline CA-28 & 65.33 & 76.43 & 70.88 & 7.67 & 7.60 & 7.64 & 46.70 & 49.13 & 48.92 \\
\hline CA-29 & 59.93 & 49.60 & 54.77 & 8.60 & 8.40 & 8.50 & 55.73 & 50.83 & 53.28 \\
\hline CA-30 & 61.43 & 65.93 & 63.68 & 8.33 & 7.80 & 8.07 & 57.07 & 52.90 & 54.99 \\
\hline CA-31 & 83.07 & 73.60 & 78.34 & 8.07 & 7.40 & 7.74 & 47.93 & 41.63 & 44.78 \\
\hline CA-32 & 81.87 & 92.40 & 87.14 & 7.27 & 6.93 & 7.10 & 53.67 & 52.20 & 52.94 \\
\hline CA-33 & 53.47 & 61.27 & 57.37 & 6.40 & 6.60 & 6.50 & 52.47 & 56.90 & 54.69 \\
\hline CA-34 & 81.47 & 83.47 & 82.47 & 6.20 & 6.75 & 6.48 & 51.00 & 54.10 & 52.55 \\
\hline CA-35 & 64.40 & 69.20 & 66.80 & 6.07 & 6.47 & 6.27 & 52.07 & 48.27 & 50.17 \\
\hline CA-36 & 66.60 & 63.93 & 65.27 & 6.13 & 6.27 & 6.20 & 51.30 & 55.33 & 53.32 \\
\hline CA-37 & 65.40 & 75.06 & 70.23 & 6.20 & 6.60 & 6.40 & 45.63 & 44.36 & 45.00 \\
\hline CA-38 & 66.60 & 64.67 & 65.64 & 6.33 & 6.40 & 6.37 & 55.33 & 53.57 & 54.45 \\
\hline CA-39 & 64.27 & 72.63 & 68.45 & 6.20 & 6.53 & 6.37 & 54.33 & 49.43 & 51.88 \\
\hline CA-40 & 66.87 & 71.77 & 69.32 & 6.13 & 5.87 & 6.00 & 53.27 & 49.37 & 51.32 \\
\hline CA-41 & 80.13 & 84.47 & 82.30 & 5.87 & 6.27 & 6.07 & 49.00 & 54.33 & 51.67 \\
\hline CA-42 & 44.53 & 42.67 & 43.60 & 5.60 & 5.81 & 5.71 & 52.67 & 51.20 & 51.94 \\
\hline CA-43 & 100.00 & 110.40 & 105.20 & 4.53 & 4.93 & 4.73 & 45.53 & 49.73 & 47.63 \\
\hline CA-44 & 80.37 & 82.83 & 81.60 & 4.13 & 4.53 & 4.33 & 55.03 & 50.13 & 52.58 \\
\hline CA-45 & 79.33 & 75.60 & 77.47 & 6.27 & 6.33 & 6.30 & 50.90 & 48.63 & 49.77 \\
\hline CA-46 & 67.07 & 62.67 & 64.87 & 6.00 & 6.20 & 6.10 & 51.53 & 53.27 & 52.40 \\
\hline CA-47 & 62.27 & 60.33 & 61.30 & 8.07 & 7.53 & 7.80 & 53.80 & 46.87 & 50.34 \\
\hline CA-48 & 54.07 & 59.67 & 56.87 & 7.20 & 7.27 & 7.24 & 50.73 & 48.37 & 49.55 \\
\hline CA-49 & 55.13 & 58.37 & 56.75 & 6.93 & 6.33 & 6.63 & 51.00 & 50.63 & 50.82 \\
\hline CA-50 & 53.47 & 50.47 & 51.97 & 7.07 & 7.27 & 7.17 & 48.53 & 48.67 & 48.60 \\
\hline CA-51 & 81.27 & 89.40 & 85.34 & 6.13 & 6.67 & 6.40 & 54.53 & 55.80 & 55.17 \\
\hline SEm \pm & 1.22 & 1.69 & 1.53 & 0.18 & 0.21 & 0.19 & 1.24 & 1.31 & 1.28 \\
\hline $\mathrm{CD}(\mathrm{P}=0.05)$ & 3.43 & 4.74 & 4.29 & 0.50 & 0.59 & 0.51 & 3.48 & 3.68 & 3.59 \\
\hline
\end{tabular}

SEm-Standard error of the mean; CD-critical difference 
Table 2. Fruits per plant, leaf chlorophyll content and fresh yield of different chilli genotypes.

\begin{tabular}{|c|c|c|c|c|c|c|c|c|c|}
\hline \multirow[t]{2}{*}{ Genotypes } & \multicolumn{3}{|c|}{ Fruits/plant } & \multicolumn{3}{|c|}{ L eaf chlorophyll (SPAD 502) } & \multicolumn{3}{|c|}{ Y ield (t/ha) } \\
\hline & $2007-08$ & $2008-09$ & Pooled & $2007-08$ & $2008-09$ & Pooled & $2007-08$ & $2008-09$ & Pooled \\
\hline CA-1 & 64.07 & 68.87 & 66.47 & 65.87 & 67.60 & 66.74 & 6.79 & 6.93 & 6.86 \\
\hline CA-2 & 47.33 & 57.27 & 52.30 & 64.00 & 60.27 & 62.14 & 5.56 & 5.92 & 5.74 \\
\hline CA-3 & 126.07 & 109.87 & 117.97 & 56.07 & 50.83 & 53.45 & 11.20 & 9.89 & 10.55 \\
\hline CA-4 & 142.67 & 123.87 & 133.27 & 58.27 & 55.03 & 56.65 & 12.24 & 10.40 & 11.32 \\
\hline CA-5 & 135.13 & 126.80 & 130.97 & 57.80 & 53.30 & 55.55 & 11.06 & 14.14 & 12.60 \\
\hline CA-6 & 102.00 & 158.13 & 130.07 & 59.90 & 52.70 & 56.30 & 9.04 & 12.34 & 10.69 \\
\hline CA-7 & 109.67 & 137.13 & 123.40 & 53.23 & 50.47 & 51.85 & 7.44 & 11.70 & 9.57 \\
\hline CA-8 & 117.07 & 122.53 & 119.80 & 53.00 & 57.63 & 55.32 & 10.19 & 10.38 & 10.29 \\
\hline CA-9 & 117.07 & 120.40 & 118.74 & 59.27 & 60.60 & 59.94 & 10.72 & 9.32 & 10.02 \\
\hline CA-10 & 89.27 & 147.27 & 118.27 & 59.87 & 61.87 & 60.87 & 8.62 & 13.47 & 11.05 \\
\hline CA-11 & 87.93 & 129.60 & 108.77 & 61.80 & 55.83 & 58.82 & 7.59 & 11.70 & 9.65 \\
\hline CA-12 & 68.67 & 99.60 & 84.14 & 57.37 & 55.70 & 56.54 & 5.68 & 8.59 & 7.14 \\
\hline CA-13 & 68.17 & 58.87 & 63.52 & 55.80 & 59.13 & 57.47 & 6.22 & 5.93 & 6.08 \\
\hline CA-14 & 64.27 & 71.60 & 67.94 & 59.83 & 60.00 & 59.92 & 6.06 & 6.48 & 6.27 \\
\hline CA-15 & 59.33 & 52.67 & 56.00 & 64.73 & 61.27 & 63.00 & 5.97 & 5.60 & 5.79 \\
\hline CA-16 & 115.67 & 146.13 & 130.90 & 64.37 & 61.33 & 62.85 & 10.34 & 13.63 & 11.99 \\
\hline CA-17 & 75.47 & 69.93 & 72.70 & 52.90 & 57.50 & 55.20 & 6.30 & 6.36 & 6.33 \\
\hline CA-18 & 95.53 & 84.27 & 89.90 & 66.00 & 64.77 & 65.39 & 8.18 & 7.38 & 7.78 \\
\hline CA-19 & 84.33 & 128.87 & 106.60 & 61.30 & 61.60 & 61.45 & 7.70 & 11.14 & 9.42 \\
\hline CA-20 & 76.13 & 54.47 & 65.30 & 59.03 & 59.30 & 59.17 & 8.91 & 6.01 & 7.46 \\
\hline CA-21 & 118.07 & 107.63 & 112.85 & 56.70 & 56.70 & 56.70 & 11.18 & 10.09 & 10.64 \\
\hline CA-22 & 113.20 & 105.73 & 109.47 & 59.60 & 62.03 & 60.82 & 10.42 & 9.61 & 10.02 \\
\hline CA-23 & 65.53 & 61.13 & 63.33 & 58.07 & 55.30 & 56.69 & 6.62 & 5.97 & 6.30 \\
\hline CA-24 & 84.73 & 110.33 & 97.53 & 54.13 & 58.43 & 56.28 & 7.22 & 10.28 & 8.75 \\
\hline CA-25 & 50.87 & 82.93 & 66.90 & 58.80 & 62.90 & 60.85 & 5.25 & 7.67 & 6.46 \\
\hline CA-26 & 112.80 & 122.87 & 117.84 & 61.90 & 60.97 & 61.44 & 9.99 & 10.44 & 10.22 \\
\hline CA-27 & 102.87 & 132.20 & 117.54 & 55.90 & 58.20 & 57.05 & 8.20 & 10.94 & 9.57 \\
\hline CA-28 & 160.67 & 141.87 & 151.27 & 61.00 & 63.10 & 62.05 & 12.69 & 10.93 & 11.81 \\
\hline CA-29 & 162.73 & 173.73 & 168.23 & 65.50 & 63.87 & 64.69 & 13.96 & 15.12 & 14.54 \\
\hline CA-30 & 154.67 & 144.27 & 149.47 & 63.37 & 64.60 & 63.99 & 13.66 & 12.32 & 12.99 \\
\hline CA-31 & 112.60 & 82.07 & 97.34 & 50.87 & 62.50 & 56.69 & 9.75 & 7.22 & 8.49 \\
\hline CA-32 & 95.67 & 84.47 & 90.07 & 54.10 & 53.10 & 53.60 & 7.62 & 7.10 & 7.36 \\
\hline CA-33 & 102.13 & 146.27 & 124.20 & 62.27 & 60.90 & 61.59 & 9.45 & 12.82 & 11.14 \\
\hline CA-34 & 116.33 & 153.00 & 134.67 & 54.73 & 58.30 & 56.52 & 10.72 & 12.07 & 11.40 \\
\hline CA-35 & 102.80 & 135.00 & 118.90 & 64.27 & 62.03 & 63.15 & 8.53 & 11.49 & 10.01 \\
\hline CA-36 & 98.80 & 146.33 & 122.57 & 59.43 & 54.37 & 56.90 & 9.01 & 13.63 & 11.32 \\
\hline CA-37 & 89.20 & 138.73 & 113.97 & 57.17 & 55.63 & 56.40 & 7.99 & 12.52 & 10.26 \\
\hline CA-38 & 87.80 & 154.67 & 121.24 & 57.37 & 59.77 & 58.57 & 7.60 & 14.13 & 10.87 \\
\hline CA-39 & 119.80 & 149.53 & 134.67 & 62.23 & 65.00 & 63.62 & 10.22 & 12.88 & 11.55 \\
\hline CA-40 & 115.13 & 170.47 & 142.80 & 55.03 & 53.57 & 54.30 & 9.89 & 14.76 & 12.33 \\
\hline CA-41 & 103.00 & 136.67 & 119.84 & 57.57 & 53.70 & 55.64 & 8.40 & 11.10 & 9.75 \\
\hline CA-42 & 86.33 & 131.93 & 109.13 & 61.67 & 64.70 & 63.19 & 8.37 & 11.79 & 10.08 \\
\hline CA-43 & 44.07 & 68.33 & 56.20 & 61.97 & 57.33 & 59.65 & 3.65 & 5.51 & 4.58 \\
\hline CA-44 & 94.00 & 135.93 & 114.97 & 58.63 & 58.00 & 58.32 & 8.53 & 11.24 & 9.89 \\
\hline CA-45 & 113.60 & 144.53 & 129.07 & 60.20 & 60.10 & 60.15 & 9.28 & 12.50 & 10.89 \\
\hline CA-46 & 109.80 & 165.73 & 137.77 & 53.37 & 56.13 & 54.75 & 9.22 & 14.29 & 11.76 \\
\hline CA-47 & 141.67 & 166.27 & 153.97 & 59.43 & 57.90 & 58.67 & 12.55 & 14.14 & 13.35 \\
\hline CA-48 & 124.60 & 164.53 & 144.57 & 56.13 & 59.20 & 57.67 & 11.93 & 14.43 & 13.18 \\
\hline CA-49 & 119.80 & 147.00 & 133.40 & 60.43 & 60.00 & 60.22 & 9.50 & 13.44 & 11.47 \\
\hline CA-50 & 121.33 & 109.33 & 115.33 & 51.77 & 49.97 & 50.87 & 10.79 & 9.44 & 10.12 \\
\hline CA-51 & 95.47 & 136.73 & 116.10 & 53.40 & 54.47 & 53.94 & 8.58 & 12.72 & 10.65 \\
\hline SEm \pm & 2.13 & 2.23 & 2.19 & 1.52 & 1.98 & 1.67 & 0.39 & 0.47 & 0.42 \\
\hline $\mathrm{CD}(\mathrm{P}=0.05)$ & 6.01 & 6.25 & 6.13 & 4.27 & 5.56 & 4.68 & 1.10 & 1.32 & 1.18 \\
\hline
\end{tabular}

SEm-Standard error of the mean; CD-critical difference 
Table 3. Ascorbic acid and capsaicin content of red fruit of different chilli genotypes.

\begin{tabular}{|c|c|c|c|c|c|c|}
\hline \multirow[t]{2}{*}{ G enotypes } & \multicolumn{3}{|c|}{ A scor bic acid (mg/100 g fresh) } & \multicolumn{3}{|c|}{ Capsaicin (\%) } \\
\hline & $2007-08$ & $2008-09$ & Pooled & $2007-08$ & $2008-09$ & Pooled \\
\hline CA-1 & 135.76 & 140.96 & 138.36 & 0.66 & 0.68 & 0.67 \\
\hline CA-2 & 108.71 & 106.14 & 107.43 & 0.93 & 0.88 & 0.91 \\
\hline CA-3 & 127.05 & 122.27 & 124.66 & 0.81 & 0.78 & 0.80 \\
\hline $\mathrm{CA}-4$ & 114.72 & 108.34 & 111.53 & 0.32 & 0.34 & 0.33 \\
\hline CA-5 & 117.80 & 119.99 & 118.90 & 0.60 & 0.57 & 0.59 \\
\hline CA-6 & 122.10 & 116.25 & 119.18 & 0.46 & 0.47 & 0.47 \\
\hline CA-7 & 136.96 & 142.35 & 139.66 & 0.34 & 0.32 & 0.33 \\
\hline CA-8 & 78.46 & 77.66 & 78.06 & 0.51 & 0.50 & 0.51 \\
\hline CA-9 & 131.93 & 134.08 & 133.01 & 0.64 & 0.63 & 0.64 \\
\hline CA-10 & 139.09 & 138.43 & 138.76 & 0.82 & 0.84 & 0.83 \\
\hline CA-11 & 91.29 & 94.53 & 92.91 & 0.74 & 0.85 & 0.80 \\
\hline CA-12 & 126.07 & 122.67 & 124.37 & 0.83 & 0.82 & 0.83 \\
\hline CA-13 & 108.71 & 109.43 & 109.07 & 0.76 & 0.76 & 0.76 \\
\hline CA-14 & 157.72 & 151.90 & 154.81 & 0.72 & 0.71 & 0.72 \\
\hline CA-15 & 73.57 & 78.20 & 75.89 & 0.80 & 0.81 & 0.81 \\
\hline CA-16 & 139.77 & 136.37 & 138.07 & 0.38 & 0.39 & 0.39 \\
\hline CA-17 & 130.88 & 134.93 & 132.91 & 1.15 & 1.10 & 1.13 \\
\hline CA-18 & 104.24 & 100.48 & 102.36 & 0.73 & 0.76 & 0.75 \\
\hline CA-19 & 150.36 & 148.15 & 149.26 & 0.64 & 0.63 & 0.64 \\
\hline CA-20 & 164.36 & 160.96 & 162.66 & 0.41 & 0.39 & 0.40 \\
\hline CA-21 & 110.17 & 106.75 & 108.46 & 0.72 & 0.73 & 0.73 \\
\hline CA-22 & 117.22 & 121.02 & 119.12 & 0.65 & 0.63 & 0.64 \\
\hline CA-23 & 124.71 & 117.92 & 121.32 & 0.84 & 0.87 & 0.86 \\
\hline CA-24 & 130.46 & 125.69 & 128.08 & 0.58 & 0.60 & 0.59 \\
\hline CA-25 & 132.93 & 128.42 & 130.68 & 0.82 & 0.80 & 0.81 \\
\hline CA-26 & 103.49 & 108.67 & 106.08 & 0.55 & 0.54 & 0.55 \\
\hline CA-27 & 169.94 & 164.48 & 167.21 & 0.79 & 0.80 & 0.80 \\
\hline CA-28 & 112.37 & 109.77 & 111.07 & 0.70 & 0.70 & 0.70 \\
\hline CA-29 & 143.76 & 142.90 & 143.33 & 0.65 & 0.63 & 0.64 \\
\hline CA-30 & 131.19 & 131.63 & 131.41 & 0.41 & 0.41 & 0.41 \\
\hline CA-31 & 118.54 & 109.86 & 114.20 & 0.78 & 0.77 & 0.78 \\
\hline CA-32 & 111.14 & 107.53 & 109.34 & 0.67 & 0.65 & 0.66 \\
\hline CA-33 & 112.96 & 119.47 & 116.22 & 0.64 & 0.67 & 0.66 \\
\hline CA-34 & 109.06 & 114.64 & 111.85 & 0.38 & 0.40 & 0.39 \\
\hline CA-35 & 131.97 & 121.88 & 126.93 & 0.74 & 0.71 & 0.73 \\
\hline CA-36 & 138.05 & 129.46 & 133.76 & 0.40 & 0.41 & 0.41 \\
\hline CA-37 & 110.13 & 118.22 & 114.18 & 0.39 & 0.40 & 0.40 \\
\hline CA-38 & 139.34 & 137.39 & 138.37 & 1.06 & 1.07 & 1.07 \\
\hline CA-39 & 140.41 & 134.09 & 137.25 & 0.57 & 0.55 & 0.56 \\
\hline CA-40 & 96.20 & 100.11 & 98.16 & 0.47 & 0.45 & 0.46 \\
\hline CA-41 & 114.20 & 111.06 & 112.63 & 0.41 & 0.40 & 0.41 \\
\hline CA-42 & 140.32 & 147.07 & 143.70 & 0.80 & 0.79 & 0.80 \\
\hline CA-43 & 105.123 & 107.76 & 106.44 & 0.73 & 0.71 & 0.72 \\
\hline CA-44 & 103.92 & 105.09 & 104.51 & 0.54 & 0.56 & 0.55 \\
\hline CA-45 & 120.92 & 119.93 & 120.43 & 0.76 & 0.78 & 0.77 \\
\hline CA-46 & 115.80 & 120.70 & 118.25 & 1.08 & 1.06 & 1.07 \\
\hline CA-47 & 124.40 & 122.43 & 123.42 & 0.37 & 0.40 & 0.39 \\
\hline CA-48 & 117.37 & 112.71 & 115.04 & 0.67 & 0.65 & 0.66 \\
\hline CA-49 & 117.28 & 113.63 & 115.46 & 0.74 & 0.76 & 0.75 \\
\hline CA-50 & 127.92 & 121.37 & 124.65 & 0.75 & 0.78 & 0.77 \\
\hline CA-51 & 136.21 & 129.68 & 132.95 & 0.54 & 0.51 & 0.53 \\
\hline SEm \pm & 5.44 & 5.51 & 4.86 & 0.02 & 0.03 & 0.02 \\
\hline $\mathrm{CD}(\mathrm{P}=0.05)$ & 15.34 & 15.48 & 13.61 & 0.06 & 0.07 & 0.06 \\
\hline
\end{tabular}

SEm-Standard error of the mean; CD-critical difference 
Table 4. White fly and yellow mites infestation in different chilli genotypes.

\begin{tabular}{|c|c|c|c|c|c|c|}
\hline \multirow[t]{2}{*}{ Genotypes } & \multicolumn{3}{|c|}{ W hite fly/plant } & \multicolumn{3}{|c|}{ Y ellow mite/leaf } \\
\hline & $2007-08$ & $2008-09$ & Pooled & $2007-08$ & $2008-09$ & Pooled \\
\hline CA-1 & 5.20 & 4.80 & 5.00 & 7.80 & 8.20 & 8.00 \\
\hline CA-2 & 2.60 & 2.80 & 2.70 & 3.80 & 4.40 & 4.10 \\
\hline CA-3 & 4.40 & 4.20 & 4.30 & 1.80 & 2.20 & 2.00 \\
\hline CA-4 & 6.20 & 5.60 & 5.90 & 0.00 & 2.00 & 1.00 \\
\hline CA-5 & 1.80 & 2.40 & 2.10 & 4.20 & 3.40 & 3.80 \\
\hline CA-6 & 1.60 & 1.20 & 1.40 & 2.20 & 3.20 & 2.70 \\
\hline CA-7 & 3.20 & 3.00 & 3.10 & 7.00 & 5.40 & 6.20 \\
\hline CA-8 & 7.00 & 7.40 & 7.20 & 10.80 & 11.00 & 10.90 \\
\hline CA-9 & 8.00 & 7.40 & 7.70 & 5.20 & 4.40 & 4.80 \\
\hline CA-10 & 3.20 & 3.60 & 3.40 & 5.00 & 6.20 & 5.60 \\
\hline CA-11 & 2.80 & 3.20 & 3.00 & 8.00 & 9.20 & 8.60 \\
\hline CA-12 & 2.00 & 1.80 & 1.90 & 14.40 & 12.80 & 13.60 \\
\hline CA-13 & 1.20 & 1.60 & 1.40 & 5.20 & 6.40 & 5.80 \\
\hline CA-14 & 2.40 & 2.00 & 2.20 & 4.80 & 5.60 & 5.20 \\
\hline CA-15 & 1.20 & 1.00 & 1.10 & 2.80 & 1.00 & 1.90 \\
\hline CA-16 & 2.20 & 1.80 & 2.00 & 2.60 & 2.40 & 2.50 \\
\hline CA-17 & 2.00 & 2.60 & 2.30 & 2.40 & 3.20 & 2.80 \\
\hline CA-18 & 1.60 & 2.00 & 1.80 & 4.20 & 0.00 & 2.10 \\
\hline CA-19 & 1.80 & 1.60 & 1.70 & 0.00 & 4.20 & 2.10 \\
\hline CA-20 & 1.00 & 1.40 & 1.20 & 3.60 & 3.20 & 3.40 \\
\hline CA-21 & 7.80 & 8.40 & 8.10 & 4.00 & 3.60 & 3.80 \\
\hline CA-22 & 7.00 & 6.60 & 6.80 & 0.00 & 0.00 & 0.00 \\
\hline CA-23 & 1.00 & 0.00 & 0.50 & 3.60 & 5.20 & 4.40 \\
\hline CA-24 & 5.20 & 5.80 & 5.50 & 0.00 & 0.00 & 0.00 \\
\hline CA-25 & 1.20 & 0.80 & 1.00 & 0.00 & 0.00 & 0.00 \\
\hline CA-26 & 2.00 & 2.60 & 2.30 & 4.40 & 2.40 & 3.40 \\
\hline CA-27 & 5.20 & 4.60 & 4.90 & 0.00 & 0.00 & 0.00 \\
\hline CA-28 & 6.60 & 5.80 & 6.20 & 4.20 & 4.60 & 4.40 \\
\hline CA-29 & 1.00 & 2.00 & 1.50 & 4.20 & 3.20 & 3.70 \\
\hline CA-30 & 3.00 & 2.40 & 2.70 & 0.00 & 0.00 & 0.00 \\
\hline CA-31 & 1.20 & 1.00 & 1.10 & 5.40 & 4.80 & 5.10 \\
\hline CA-32 & 2.20 & 2.40 & 2.30 & 5.20 & 4.20 & 4.70 \\
\hline CA-33 & 2.40 & 2.00 & 2.20 & 2.60 & 1.00 & 1.80 \\
\hline CA-34 & 5.00 & 5.60 & 5.30 & 0.00 & 4.40 & 2.20 \\
\hline CA-35 & 1.00 & 0.60 & 0.80 & 0.00 & 0.00 & 0.00 \\
\hline CA-36 & 4.00 & 3.80 & 3.90 & 3.40 & 4.20 & 3.80 \\
\hline CA-37 & 3.40 & 3.60 & 3.50 & 4.80 & 4.00 & 4.40 \\
\hline CA-38 & 4.60 & 4.00 & 4.30 & 2.40 & 3.80 & 3.10 \\
\hline CA-39 & 3.00 & 3.80 & 3.40 & 3.80 & 0.00 & 1.90 \\
\hline CA-40 & 3.00 & 3.20 & 3.10 & 5.20 & 4.00 & 4.60 \\
\hline CA-41 & 5.80 & 3.60 & 4.70 & 0.00 & 2.20 & 1.10 \\
\hline CA-42 & 4.60 & 4.00 & 4.30 & 4.20 & 3.40 & 3.80 \\
\hline CA-43 & 0.00 & 0.80 & 0.40 & 6.60 & 3.00 & 4.80 \\
\hline CA-44 & 6.80 & 5.40 & 6.10 & 1.20 & 2.20 & 1.70 \\
\hline CA-45 & 0.00 & 0.00 & 0.00 & 2.60 & 2.00 & 2.30 \\
\hline CA-46 & 2.20 & 1.80 & 2.00 & 2.80 & 0.00 & 1.40 \\
\hline CA-47 & 3.60 & 3.60 & 3.60 & 2.20 & 4.20 & 3.20 \\
\hline CA-48 & 4.60 & 4.20 & 4.40 & 4.00 & 3.20 & 3.60 \\
\hline CA-49 & 2.40 & 3.20 & 2.80 & 2.20 & 2.40 & 2.30 \\
\hline CA-50 & 4.20 & 3.60 & 3.90 & 1.80 & 0.00 & 0.90 \\
\hline CA-51 & 5.00 & 4.20 & 4.60 & 3.60 & 2.80 & 3.20 \\
\hline SEm \pm & 0.07 & 0.08 & 0.07 & 0.10 & 0.11 & 0.10 \\
\hline $\mathrm{CD}(\mathrm{P}=0.05)$ & 0.20 & 0.22 & 0.20 & 0.28 & 0.30 & 0.28 \\
\hline
\end{tabular}

SEm-Standard error of the mean; CD-critical difference 
was lowest $(75.89 \mathrm{mg} / 100 \mathrm{~g}$ fresh) was observed in CA15. Deshpande and Anand (1988) estimated ascorbic acid content ranged from $58.7 \mathrm{mg}$ to $192.1 \mathrm{mg} / 100 \mathrm{~g}$ fresh which was more or less similar with the findings of the present experiment. The significantly highest capsaicin in red fruit was recorded in genotype CA-17 $(1.13 \%)$. More than $1 \%$ capsaicin content was recorded in genotypes CA-38 $(1.07 \%)$ and CA-46 (1.07\%). The lowest capsaicin content in red fruit was recorded in genotype CA-4 (0.33).

W hite fly and yellow mite incidence: Genotype CA-45 was almost free from white fly incidence and lower white fly incidence was also recorded in genotype CA - $43(0.40 /$ plant) followed by CA- 23 (0.50/plant) and CA -21 (8.10/ plant) was found most susceptible to white fly incidence (Table 4). Among the different genotypes CA - 22, CA 24, CA - 25, CA - 27 and CA - 30 were free from mite infestation where as CA - 13 (13.60/leaf) was most susceptible to yellow mite incidence.

\section{Conclusion}

The present study concluded that the genotype CA-29, CA-47 and CA-48 may be adopted for cultivation in the terai zone of West Bengal for their higher yield and moderate quality characters. However, incidence of white fly population was almost nil in CA- 45 followed by CA 43 (0.40/plant). Genotypes CA - 22, CA - 24, CA - 25, CA -27 and $C A-30$ were free from mite infestation.

\section{REFERENCES}

Amin, P.W. (1979). Leaf curl diseases of chilli pepper in Maharastra, India. PANS, 25: 131-134.

Awate, B.G., Gandhale, D.N., Naik, L.M. and Patil, A.S. (1981). Chemical control of mite Polyphagotarsonemus latus on chilli. Indian J ournal of Plant Protection, 30: 193.

Brown, J.K. (1994). Current status of Bemisia tabaci as a plant pest and virus vector in agroecosystems worldwide. FAO Plant Protection Bulletin, 42 (12): 332

Chaudhary, B.S. and Samadia, D.K. (2004). Variability and character association in chilli landraces and genetypes under arid environment. Indian J ournal of Horticulture, 61 (2): 132-136.

Deb, P., Datta, S. and Paul, P.K. (2008). Studies on performance of in chilli (Capsicum annuum L.) genotypes under terai zone of West Bengal. Indian J ournal of H orticulture, 65 (3): 353-355.
Deshpande, A.A. and Anand, N. (1988). Chilli breeding works at Indian Institute of Horticultural Research, Bangalore. Proceeding of National Seminar on chillies, Ginger and Turmeric, held during 11-12 January, 1988 at Hyderabad, pp. 17-24.

Gogate, S.M., Patel, R.K., Patel, M..J. and Patel, J.A. (2006). Studied on the genetic divergence in chilli (C apsicum annuum Var. Longum (D.C.) Sendt.). Vegetable Science, 33 (1): 2125

Gomez K.A. and Gomez A.A. (1984) . Statistical Procedures for Agricultural Research. John Wiley and Sons Publication ( $2^{\text {nd }}$ Edition), pp. 20-28.

Hundal, J.S. and Khurana, D.S. (1988). Heterosis potential in chillies (Capsicum annuum L.). Proceeding of National Seminar on chillies, Ginger and Turmeric, held on 11-12 January, 1988 at Hyderabad, pp. 33-37.

Jagadeesha, R.C., Prabhu, S.T. and Tatagar, M. (2000). Genetic analysis of resistance to thrips and mites in chilli (Capsicum annuum L.). Proceedings of $5^{\text {th }}$ International Solanaceous Confer ence, University Nizmegan, Netharlands, $22^{\text {nd }}-29^{\text {th }}$ March, 2000.

Khurana, D.S., Singh,P. and Hundal, J.S. (2003). Studies on genetic diversity for growth, yield and quality traits in chilli (Capsicum annuum L.). Indian J ournal of H orticulture, 60 (3): $227-282$.

Mondal, B. and Mondal, P. (2012). Ecofriendly pest management practices for leaf curl complex of chilli (Capsicum annuum L.). J ournal of Biopest, 5: 115-118.

Othman, A. K., Shuaib M.O.S. and Sattar, M.H.A. (2002). Survey for host plants of whitefly, Bemisia tabaci (Gnn.) in Abyan and Tuban Delta at southern coastal plain. U niversity of Aden J ournal of Natural and Applied Sciences, 6 (3): 497504.

Prasad, B.C. and Reddy, K.M. and sadashiva, A.T. (2003) Heterosis in chilli (Capsicum annuum L.). Indian J ournal of Horticulture, 60 (1): 69-74.

Ranganna, S. (2001). Analysis and quality control for fruits and vegetable products. Tata Mc Grawhill Publication. $2^{\text {nd }}$ Edition, New Delhi, India, pp.110-112

Sadasivam, S. and Manickam, A. (1996). Ascorbic Acid. In: Biochemical Methods. New Age International Publishers, ( $2^{\text {nd }}$ Edition), Ned Delhi, India,pp. 185-186

Saikia, A.K. and Muniyappa, V. (1989). Epidemilogy and control of tomato leaf curl in southern India. Tropical Agriculture, 66: 350-354.

Thresh, J.M. (1974). Temporal patterns in virus spread. Annual Review of Phytopathology, 12: 111-128. 